\section{REVIEW OF HOSPITAL PATIENTS WITH ALT>1000 IU/L}

A McDonough, Z Galvin, J Ryan, M T O'Neill, H Fitzpatrick, S Stewart Centre for Liver Disease, Mater Misericordiae University Hospital, Dublin, Ireland

10.1136/gutjnl-2013-305143.86

Introduction There are many potential causes for an ALT $>1000 \mathrm{IU} / \mathrm{L}$, the commonest being ischaemia, drug induced liver injury (DILI) and viral hepatitis. There are however many other potential causes, and, more recently, acute endemic hepatitis $\mathrm{E}(\mathrm{HEV})$ has been identified in some of these patients.

Aims/Background Our aim was to establish the causes of ALT $>1000 \mathrm{IU} / \mathrm{L}$ in patients in a large teaching hospital. We also wanted to determine the number of patients in whom a cause was not found, and how many of these had been checked for HEV.

Method All ALT values over a two-year period (2010/11) were examined. Those with an ALT $>1000$ IU/L were identified and their investigations/notes reviewed. All data was annonymised and recorded in a dedicated electronic database.

Results 182 patients (57\% male and 43\% female) with an ALT $>1000 \mathrm{IU} / \mathrm{L}$ were identified. The mean age for males versus females was $47+/-18$ years versus $62+/-18$ years $(p=0.001)$. The most common causes of an ALT $>1000 \mathrm{IU} / \mathrm{L}$ were ischaemic hepatitis, $(n=111,(61 \%))$, DILI, $(n=30,(16.5 \%))$ and viral hepatitis, $(n=22,(12.1 \%))$. The remaining causes included choledocholithiasis $(n=8)$ and autoimmune hepatitis $(n=3)$. No cause was identified for 8 patients. Of these, none had HEV IgM checked. $35.7 \%(n=65)$ died during this admission, $54.9 \%$ $(n=100)$ were discharged home and 9.3\% (17) were not admitted.

Conclusion This review confirms that ischaemic liver injury is the commonest cause of ALT $>1000$ IU/L in hospital inpatients. Ischaemia, DILI and viral hepatitis account for almost $90 \%$ of presentations. Only $5 \%$ of patients had no aetiology identified.
This number may be significantly lower if all had had HEV IgM checked. 